\title{
Genetic loci mapping associated with maize kernel number per ear based on a recombinant inbred line population grown under different nitrogen regimes
}

\author{
X.H. Liu', S.L. He ${ }^{2}$, Z.P. Zheng ${ }^{2}$, Z.B. Tan ${ }^{3}$ Z. $\mathrm{Li}^{2}$ and C. $\mathrm{He}^{2}$ \\ ${ }^{1}$ Key Laboratory of Southwest China Wildlife Resources Conservation, \\ Ministry of Education, College of Life Sciences, \\ China West Normal University, Nanchong, P.R. China \\ ${ }^{2}$ Nanchong Institute of Agricultural Sciences, Nanchong, P.R. China \\ ${ }^{3}$ Beijing IPE Bio-Technology Co., Ltd., Beijing, P.R. China \\ Corresponding author: Z.P. Zheng \\ E-mail: 1xhxhsfdx@yahoo.com.cn
}

Genet. Mol. Res. 10 (4): 3267-3274 (2011)

Received March 15, 2011

Accepted September 28, 2011

Published December 22, 2011

DOI http://dx.doi.org/10.4238/2011.December.22.5

\begin{abstract}
Kernel number per ear (KNE) is one of the most important yield-related agronomic traits in maize (Zea mays). To clarify its genetic basis, we made a quantitative trait locus (QTL) analysis of KNE in a recombinant inbred line population derived from lines Mo17 and Huangzao4, under two nitrogen $(\mathrm{N})$ regimes. Seven QTLs, on chromosomes 4, 6 and 9, were mapped under the high $\mathrm{N}$ regime, which explained phenotypic variation ranging from 5.03 to $15.49 \%$. Under the low N regime, three QTLs were located on chromosomes 6 and 9, which accounted for phenotypic variation ranging from 8.54 to $12.21 \%$. These QTLs had different mapping intervals to their nearest markers, ranging from 0 to $16.5 \mathrm{cM}$. According to the chromosome positions and genetic effects of these QTLs, only seven QTLs for KNE were identified in our experiment, out of which three were found under both $\mathrm{N}$ regimes, on chromosomes 6 (one) and 9 (two); the other four
\end{abstract}


were mapped only under the high $\mathrm{N}$ regime, on chromosomes 4 (three) and 6 (one). This information could be useful for developing marker-assisted selection in maize-breeding projects.

Key words: Maize (Zea mays); Kernel number per ear; Nitrogen; Quantitative trait locus

\section{INTRODUCTION}

Kernel number per ear (KNE) is one of the most important agronomic traits in maize (Zea mays L.) related to yield, but in breeding practice, maize germplasm presenting desirable KNE is scarce. Traditional breeding efforts for improving the trait depend on a lot of field testing, and accurate field evaluation is often laborious and time-consuming (Duvick et al., 2004). Whereas, markerassisted selection (MAS), which enables breeders to distinguish genotypes directly, is a better approach for developing desirable KNE germplasm, and quantitative trait locus (QTL) analysis can help us to understand the genetic basis of a trait, enabling its use in MAS (Tuberosa et al., 2002).

Previous studies on QTL mapping of maize ear-related traits focused on these aspects, including yield per plant (Huang et al., 2010), 100-kernel weight (Guo et al., 2008), ear weigh (Sabadin et al., 2008), row number per ear (Lu et al., 2006), and kernel number per row (Li et al., 2010). For the trait KNE, although there are also some reports published in the literature (Ribaut et al., 1997; Agrama et al., 1999; Frova et al., 1999; Gallais and Hirel, 2004; Xiao et al., 2005; Prasannaet al., 2009), different parental lines may lead to different results in QTL number, chromosomal location or genetic effect. For example, using X178 and B73 as parental inbred lines, Xiao et al. (2005) identified six QTLs for KNE on chromosomes 1 (three), 3 (one) and 9 (two), but in the report by Agrama et al. (1999), using B73 and G79 as mapping parents, there were four QTLs on chromosomes 1 (one), 6 (one), 7 (one), and 9 (one). Thus, it is necessary to use other parental lines to map the QTLs associated with KNE in maize. These different results may also be due to sampling of the segregation populations used for QTL identification, and the populations in previous studies were focused on $\mathrm{F}_{2}$ possessing the deficiency named temporality (Ribaut et al., 1997; Agrama et al., 1999; Xiao et al., 2005). Alternatively, a recombinant inbred line (RIL) population is immortal and can be utilized repeatedly, owing to homogenous genotypes forming segregating populations (Pilet et al., 2001). However, RIL populations have been rarely used to map the QTLs for KNE, despite being frequently applied to other agronomic traits of maize (Tang et al., 2010; Trachsel et al., 2010). Therefore, it is useful to carry out the studies on QTL mapping for KNE using a RIL population in maize.

Different environmental conditions can also lead to different results in QTL number, position or genetic effect. For instance, Agrama et al. (1999) identified four QTLs on chromosomes 1, 4, 6, and 9 under high nitrogen $(\mathrm{N})$ environments, while under $\mathrm{N}$ stress conditions, they detected four QTLs distributed on chromosomes 1, 3, 6, and 9. Among the ecological conditions influencing maize growth and development, $\mathrm{N}$ content in the soil is a very important one. Currently, most maize in the world is grown under N-deficient conditions because of low $\mathrm{N}$ use efficiency, limited availability of fertilizer or low purchasing power of farmers (Ribaut et al., 2007). From the literature, different $\mathrm{N}$ regimes have been frequently used to detect QTLs in plant such as rice (Oryza sativa L.) (Lian et al., 2005), maize (Liu et al., 2008) and wheat (Triticum aestivum L.) (An et al., 2006), but studies on QTL mapping for maize $\mathrm{KNE}$ under different $\mathrm{N}$ regimes have been rarely reported (Agrama et al., 1999). Accordingly, 
it is meaningful that different $\mathrm{N}$ regimes that affect KNE in maize are used for QTL mapping. Therefore, an RIL population derived from the two parental lines Mo17 and Huangzao4 was used for identifying the QTL(s) controlling KNE under two N regimes, with the aim of 1) determining the genetic basis of the trait KNE more clearly in maize, and 2) finding some molecular markers co-segregated with the genetic loci controlling KNE, which can be used for MAS in maize breeding projects.

\section{MATERIAL AND METHODS}

\section{Plant materials}

The experimental materials used in this study included the inbred lines Mo17 and Huangzao4, their $\mathrm{F}_{1}$ hybrid, and a segregating population consisting of 239 RILs derived from this cross. Mo17 and Huangzao4 are the representative lines of Lancaster (USA) and Tansipingtou heterotic group (P.R. China), respectively; these inbreds are characterized by wide adaptability, high combining ability and high resistance to pathogens. Presently the two inbreds are widely applied in producing elite hybrids.

\section{Field experiments and statistical analyses}

All entries were sown at the experiment field of Nanchong Institute of Agricultural Sciences, Nanchong City, P.R. China, in a randomized block design, where each plot contained 15 plants. Among the six replicates of each genotype, three were under high $\mathrm{N}$ regime (HNR) by applying urea at a level of $300 \mathrm{~kg} / \mathrm{ha}$, and the other three were under low $\mathrm{N}$ regime (LNR) with no appended $\mathrm{N}$ fertilizer. The average content of total $\mathrm{N}$ and alkaline hydrolyzed $\mathrm{N}$ in 30-cm depth soil were 0.092 and $0.000056 \%$, respectively.

During harvest, the middle eight plants for each replicate of the 242 genotypes were taken individually to determine phenotypic values for the trait KNE. Then, the SPSS11.5 software (www.spss.com) was used to perform descriptive statistics, analysis of variance (ANOVA), and correlation analysis for the trait of parental lines, $\mathrm{F}_{1}$ hybrid and RIL population.

\section{QTL identification}

Based on the phenotypic data of the RIL population under the two N regimes and the genetic map, consisting of 100 simple sequence repeat (SSR) markers, and covering a mapping distance of $1421.5 \mathrm{cM}$ (Liu et al., 2009), the QTL(s) associated with KNE were detected by composite interval mapping (CIM) using the Windows QTL Cartographer 2.5 software (Wang et al., 2010). Control parameters included standard CIM model, 5 control markers, 10$\mathrm{cM}$ window size, $2.0 \mathrm{cM}$ as walk speed, and forward regression method. The $\log _{10}$ of odds ratio (LOD) value for the QTL significance threshold was determined by 1000-time permutation test based on phenotypic data of the trait $(\alpha=0.05)$ (Doerge and Churchill, 1996). The QTL(s) with an LOD value greater than the threshold were presented, and their chromosomal position, genetic effects, and percentage of phenotypic variance explained by them were estimated at the significant LOD peak in the region, then, the identified QTL(s) were mapped using the Mapchart 2.1 software (Voorrips, 2002). 


\section{RESULTS}

\section{Phenotypic observation and statistical analysis}

The data showed that the tested genotypes had different phenotypic values for the trait KNE. For the three entries including Mo17, Huangzao4 and $\mathrm{F}_{1}$ hybrid, the $\mathrm{F}_{1}$ hybrid had much higher KNE values than the others under the two N regimes (Table 1), probably due to heterosis. Under HNR, Huangzao4 presented higher values than Mo17, while under LNR, the result was opposite. The results for the RIL population are displayed in Table 1. The two parameters, including standard deviation (SD) and skewness, possessed higher values under HNR than those under LNR, while the others had opposite results. From the frequency distribution graphs of the RIL population under two $\mathrm{N}$ regimes (Figure 1), it was found that the two sets of data agreed with a normal distribution; this provided evidence that KNE was a quantitative trait controlled by multiple genes in maize.

Table 1. Descriptive statistics of parental lines, $\mathrm{F}_{1}$ hybrid and recombinant inbred line (RIL) population derived from the cross Mo17 $\times$ Huangzao4 for KNE under two nitrogen (N) regimes.

\begin{tabular}{|c|c|c|c|c|c|c|c|c|}
\hline \multirow[t]{2}{*}{$\mathrm{N}$ regimes } & \multirow{2}{*}{$\frac{\text { Mo17 }}{(\text { Mean } \pm \text { SD })}$} & \multirow{2}{*}{$\frac{\text { Huangzao4 }}{(\text { Mean } \pm \text { SD) }}$} & \multirow{2}{*}{$\frac{\mathrm{F}_{1} \text { hybrid }}{(\text { Mean } \pm \mathrm{SD})}$} & \multicolumn{5}{|c|}{ RIL population } \\
\hline & & & & Minimum & Maximum & $($ Mean $\pm \mathrm{SD})$ & Skewness & Kurtosis \\
\hline . & $169.53 \pm$ & $241.97 \pm$ & 575.39 & 43.6 & 478.32 & 265. & -0 & -0.139 \\
\hline LNR & $226.03 \pm 63.17$ & $180.83 \pm 72.84$ & $554.59 \pm 34.32$ & 71.20 & 558.99 & $284.69 \pm 78.38$ & -0.142 & 0.379 \\
\hline
\end{tabular}

$\mathrm{SD}=$ standard deviation. For abbreviations, see legend to Figure 1.

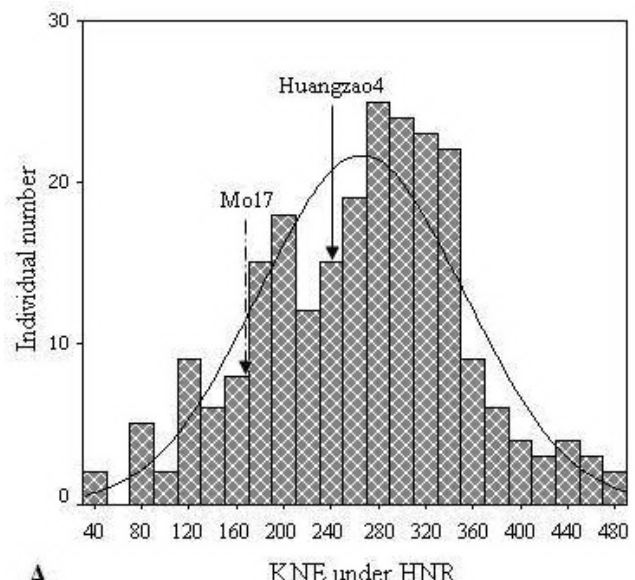

A

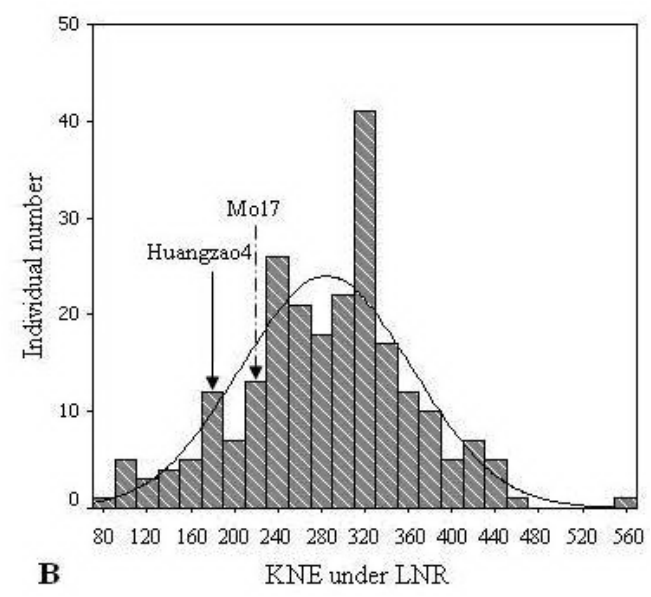

Figure 1. Frequency distribution graphs of the recombinant inbred line population derived from Mo17 $\times$ Huangzao4 for kernel number per year (KNE) under two N regimes. A. KNE under HNR. B. KNE under LNR. The means of parental lines (Mo17 and Huangzao4) are indicated by arrows. HNR and LNR $=$ high and low nitrogen regimes, respectively.

In addition, a complete ANOVA model was used to analyze the RIL population, and the results showed that the differences in different $\mathrm{N}$ regimes and different genotypes were significant at a 0.01 probability level, but the difference of interactions between them is not significant at a 0.05 probability level (Table 2 ). Nevertheless, the two sets of data for the RIL population, ob- 
tained under two $\mathrm{N}$ regimes, indicated a positive correlation at a 0.01 probability level $(\mathrm{r}=0.778)$.

\begin{tabular}{|c|c|c|c|c|}
\hline Source of variation & d.f. & SS & MS & $F$ \\
\hline Treatment combinations & 471 & 9761306.97 & 20724.64 & $9.42 * *$ \\
\hline Different $\mathrm{N}$ regimes & 1 & 125146.50 & 125146.50 & $56.88^{* *}$ \\
\hline Different genotypes & 235 & 9064682.82 & 38573.12 & $17.53 * *$ \\
\hline Different $\mathrm{N}$ regime $\times$ different genotypes & 235 & 571477.66 & 2431.82 & 1.11 \\
\hline Experimental error & 944 & 2077012.10 & 2200.23 & \\
\hline Total variation & 1415 & 11838319.07 & & \\
\hline
\end{tabular}

\section{QTL identification}

The results of the permutation test demonstrated that the LOD values of QTL significance threshold should be set at 2.76 and 2.63 for HNR and LNR, respectively. Based on the LOD threshold, seven QTLs were identified under HNR, with 3 (Qhn4a, Qhn $4 b$ and $Q h n 4 c), 2$ (Qhn6a and $Q h n 6 b)$ and 2 (Qhn9a and Qhn9b) on chromosomes 4, 6 and 9, respectively (Figure 2). These QTLs could account for 5.03 to $15.49 \%$ of phenotypic variance (Table 3). Due to negative additive effects, the five QTLs on chromosomes 4 (Qhn4a, Qhn $4 b, Q h n 4 c)$ and 6 (Qhn6a and Qhn6b) could make KNE decrease -22.44, -24.11, -30.78, -23.62, and -34.24, respectively; while the two on chromosome 9 ( $Q h n 9 a$ and $Q h n 9 b)$ could increase KNE about 29.97 and 33.50, respectively, due to positive additive effects.
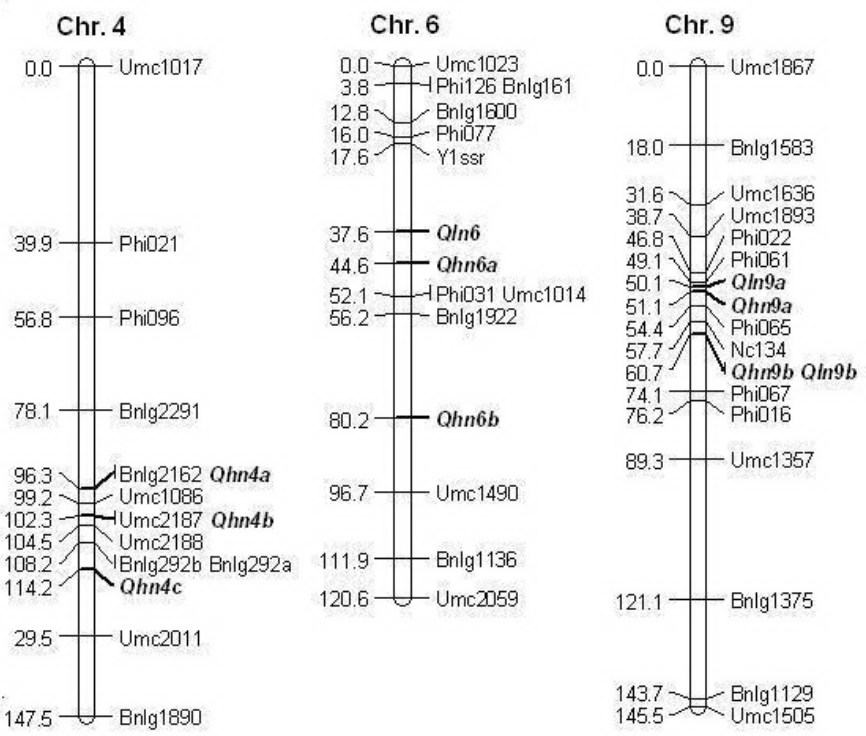

Figure 2. Chromosomal positions of the QTLs for KNE identified using the RIL population derived from Mo17 $\times$ Huangzao4 under two N regimes. Qhn4a, Qhn4b, Qhn4c, Qhn6a, Qhn6b, Qhn9a, and Qhn9b were detected under HNR, while $Q \ln 6, Q \ln 9 a$ and $Q \ln 9 b$ were identified under LNR. 


\begin{tabular}{|c|c|c|c|c|c|c|c|c|}
\hline$\overline{\mathrm{N} \text { regime }}$ & Chr. & QTL & Position (cM) & $\begin{array}{l}\text { Linked } \\
\text { markers }\end{array}$ & $\begin{array}{c}\text { Mapping } \\
\text { interval (cM) }\end{array}$ & LOD & $R^{2}(\%)$ & $\begin{array}{l}\text { Additive } \\
\text { effects }\end{array}$ \\
\hline \multirow{7}{*}{ High N } & 4 & $Q h n 4 a$ & 96.3 & Bnlg2162 & 0 & 2.89 & 5.03 & -22.44 \\
\hline & & Qhn $4 b$ & 102.3 & Umc2187 & 0 & 3.52 & 5.88 & -24.11 \\
\hline & & Qhn $4 c$ & 114.2 & Bnlg292a & 6.0 & 4.38 & 11.10 & -30.78 \\
\hline & 6 & Qhn6a & 44.6 & Phi031 & 7.5 & 2.78 & 7.28 & -23.62 \\
\hline & & Qhn6b & 80.2 & Umc1490 & 16.5 & 2.82 & 15.49 & -34.24 \\
\hline & 9 & Qhn9a & 51.1 & Phi061 & 2.0 & 5.64 & 11.73 & 29.97 \\
\hline & & Qhn $9 b$ & 60.7 & $\mathrm{Nc} 134$ & 3.0 & 6.15 & 14.86 & 33.50 \\
\hline \multirow[t]{3}{*}{ Low N } & 6 & $Q \ln 6$ & 37.6 & Phi031 & 14.5 & 2.70 & 10.50 & -25.56 \\
\hline & 9 & $Q \ln 9 a$ & 50.1 & Phi061 & 1.0 & 4.07 & 8.54 & 23.17 \\
\hline & & $Q \ln 9 b$ & 60.7 & $\mathrm{Nc} 134$ & 3.0 & 4.56 & 12.21 & 27.46 \\
\hline
\end{tabular}

Mapping interval $=$ between QTL and linker marker. $\mathrm{LOD}=\log _{10}$ of odds ratio. $R^{2}(\%)=$ percentage of phenotypic variation explained by QTL.

Under LNR, there were three QTLs detected on chromosomes $6(Q \ln 6)$ and $9(Q \ln 9 a$ and $Q \ln 9 b)$, linked with Phi031, Phi061 and Nc134, respectively, with a mapping distance of 1.0-14.5 cM between these QTLs and their linked markers. The three QTLs could explain 10.5, 8.54 and $12.21 \%$ of phenotypic variance, respectively. Of which, the QTL mapped on chromosome 6 had negative additive value and could decrease KNE by -25.56 ; while the two located on chromosome 9 could increase KNE by about 23.17 and 27.46, respectively, owing to positive additive effects.

According to the chromosomal positions and genetic effects of these QTLs (Figure 2 and Table 3), it was concluded that $Q h n 6 a$ and $Q \ln 6, Q h n 9 a$ and $Q \ln 9 a$, and $Q h n 9 b$ and $Q \ln 9 b$ were the same QTLs, respectively; therefore, in this study, only seven QTLs were identified, among them three were detected under both $\mathrm{N}$ regimes, and four were located only under HNR.

\section{DISCUSSION}

KNE is a very important agronomic trait in maize breeding programs, related to yield. To realize its genetic basis, in this study, an RIL population derived from a cross between Mo17 and Huangzao4 was used to detect the QTLs associated with KNE under two N regimes, and a total of seven QTLs were identified on chromosomes 4 (three), 6 (two) and 9 (two).

It is worth noting that QTLs for KNE have also been detected by other researchers. However, the previous segregating populations were given for $\mathrm{F}_{2: 3}$ families (Ribaut et al., 1997; Agrama et al., 1999; Xiao et al., 2005); this kind of population is temporary and cannot be reused, because no continued plants are used for phenotypic and genetic analysis (Pilet et al., 2001). But the RIL population, as used in our study, is immortal and could be utilized in different regions and times, similar to previous reports (Tang et al., 2010; Trachsel et al., 2010). Moreover, previous ecological conditions designed for QTL mapping controlling KNE were mainly concentrated on water-content differences in soil (Ribaut et al., 1997; Frova et al., 1999; Xiao et al., 2005; Prasanna et al., 2009), whereas different N regimes were rarely used, although it is a very important one affecting KNE. Furthermore, the QTLs identified in our study were different from the previous results, such as QTL number, position and genetic effect, which could probably be explained by different parental lines, segregating populations, ecological conditions or molecular markers.

Although similar N differences in soil were also used by Agrama et al. (1999) to map 
QTLs for KNE, our studies were still different from theirs in many aspects. Take the positions and number of mapped QTLs as an example, for chromosomes 1 and 7, one and one QTLs were mapped in their experiment, respectively, while in ours, no QTLs were found. For chromosome 4, no QTLs were mapped in the study by Agrama et al. (1999), but in our study we found three QTLs. For chromosome 6, only one QTL was found under LNR in their experiment, but in our study, two QTLs were mapped, among which, only one under HNR and the other under both N regimes. For chromosome 9, only one QTL was identified under HNR in their study, whereas in ours, two QTLs were simultaneously detected under both $\mathrm{N}$ regimes. These differences between the two results could be due to different parental lines, segregation population or genetic map.

As is well known that maize KNE is an agronomic trait related to row number per ear (RNE) and grain number per row (GNR); high RNE and GNR determine high KNE. In our previous experiment on QTL mapping for RNE and GNR (Liu et al., 2010), three QTLs for RNE were identified, two and one on chromosomes 4 and 9, respectively; five QTLs for GNR were mapped on chromosomes 1 (one), 2 (one), 6 (two), and 9 (one). Comparing the two studies in QTL position, all these QTLs had different chromosomal positions, except for the locus bin6.07, including one QTL for GNR and another QTL for KNE. However, most QTLs were focused on chromosomes 4,6 and 9. For additive effects, all the QTLs mapped on chromosomes 9 possessed positive values, while the other QTLs identified on chromosomes 1, 2, 4, and 6 had negative values, except for the one affecting RNE on chromosome 4.

Furthermore, among the seven QTLs identified in this present study, the two on chromosome 4 ( $Q h n 4 a$ and $Q h n 4 b)$ were co-located in proximity to SSR markers Bnlg2162 and Umc2187, respectively, with a mapping distance of $0 \mathrm{cM}$. This suggested that the linked markers are probably co-segregating with the genetic loci controlling KNE, thus, they could be considered for MAS in maize breeding programs. The other QTLs were placed far from their linked markers, ranging from 1.0 to $16.5 \mathrm{cM}$. In this respect, we could add other molecular markers to the given chromosomal regions to map these QTLs more finely, and further study is in progress based on the constructed RIL population and genetic map.

In summary, an RIL population, derived from the two parental lines Mo17 and Huangzao4, was used to map the QTLs associated with KNE under two $\mathrm{N}$ regimes in our study. As a result, seven QTLs were mapped on chromosomes 4 (three), 6 (two) and 9 (two) under HNR, which could explain phenotypic variances of 5.03 to $15.49 \%$, while under LNR, only three QTLs were located on chromosomes 6 (one) and 9 (two), accounting for total phenotypic variances of 8.54 to $12.21 \%$. All the identified QTLs had negative additive effects, except for these detected under chromosome 9. But from QTL position and effect, it was concluded that only seven QTLs were identified, among which three were found under both $\mathrm{N}$ regimes, one and two on chromosomes 6 and 9, respectively, while the other four were located only under HNR, three on chromosome 4 and one on chromosome 6. These QTLs were near to their linked markers, with a mapping distance of $0-16.5 \mathrm{cM}$. These results are beneficial for understanding the genetic basis of KNE and developing MAS in maize breeding projects.

\section{ACKNOWLEDGMENTS}

Research supported by the Chinese Sichuan Science Foundation for Young Scientists (\#2007q14-029) and the Scientific Research Fund of Sichuan Provincial Education Department (\#08ZA020). 


\section{REFERENCES}

Agrama HAS, Zakaria AG, Said FB and Tuinstra M (1999). Identification of quantitative trait loci for nitrogen use efficiency in maize. Mol. Breed. 5: 187-195.

An D, Su J, Liu Q, Zhu Y, et al. (2006). Mapping QTLs for nitrogen uptake in relation to the early growth of wheat (Triticum aestivum L.). Plant Soil 284: 73-84.

Doerge RW and Churchill GA (1996). Permutation tests for multiple loci affecting a quantitative character. Genetics 142: 285-294.

Duvick DN, Smith JSC and Cooper M (2004). Long-term selection in a commercial hybrid maize breeding program. Plant Breed. Rev. 24: 109-151.

Frova C, Krajewski P, di Fonzo N, Villa M, et al. (1999). Genetic analysis of drought tolerance in maize by molecular markers I. Yield components. Theor. Appl. Genet. 99: 280-288.

Gallais A and Hirel B (2004). An approach to the genetics of nitrogen use efficiency in maize. J. Exp. Bot. 55: 295-306.

Guo J, Su G, Zhang J and Wang G (2008). Genetic analysis and QTL mapping of maize yield and associate agronomic traits under semi-arid land condition. Afr. J. Biotechnol. 7: 1829-1838.

Huang YF, Madur D, Combes V, Ky CL, et al. (2010). The genetic architecture of grain yield and related traits in Zea maize L. revealed by comparing intermated and conventional populations. Genetics 186: 395-404.

Li M, Guo X, Zhang M, Wang X, et al. (2010). Mapping QTLs for grain yield and yield components under high and low phosphorus treatments in maize (Zea mays L.). Plant Sci. 178: 454-462.

Lian X, Xing Y, Yan H, Xu C, et al. (2005). QTLs for low nitrogen tolerance at seedling stage identified using a recombinant inbred line population derived from an elite rice hybrid. Theor. Appl. Genet. 112: 85-96.

Liu XH, Tan ZB and Rong TZ (2009). Molecular mapping of a major QTL conferring resistance to SCMV based on immortal RIL population in maize. Euphytica 167: 229-235.

Liu XH, He SL, Zheng ZP, Huang YB, et al. (2010). QTL identification for row number per ear and grain number per row in maize. Maydica 55: 127-133.

Liu ZH, Xie HL, Tian GW, Chen SJ, et al. (2008). QTL mapping of nutrient components in maize kernels under low nitrogen conditions. Plant Breed. 127: 279-285.

Lu GH, Tang JH, Yan JB, Ma XQ, et al. (2006). Quantitative trait loci mapping of maize yield and its components under different water treatments at flowering time. J. Integr. Plant Biol. 48: 1233-1243.

Pilet ML, Duplan G, Archipiano H, Barret P, et al. (2001). Stability of QTL for field resistance to blackleg across two genetic backgrounds in oilseed rape. Crop Sci. 41: 197-205.

Prasanna BM, Beiki AH, Sekhar JC, Srinivas A, et al. (2009). Mapping QTLs for component traits influencing drought stress tolerance of maize (Zea mays L) in India. J. Plant Biochem. Biotechnol. 18: 151-160.

Ribaut JM, Jiang C, Gonzalez-de-Leon D, Edmeades GO, et al. (1997). Identification of quantitative trait loci under drought conditions in tropical maize. 2. Yield components and marker-assisted selection strategies. Theor. Appl. Genet. 94: 887-896.

Ribaut JM, Fracheboud Y, Monneveux P, Banziger M, et al. (2007). Quantitative trait loci for yield and correlated traits under high and low soil nitrogen conditions in tropical maize. Mol. Breed. 20: 15-29.

Sabadin PK, Souza CL Jr, Souza AP and Garcia AAF (2008). QTL mapping for yield components in a tropical maize population using microsatellite markers. Hereditas 145: 194-203.

Tang J, Yan J, Ma X, Teng W, et al. (2010). Dissection of the genetic basis of heterosis in an elite maize hybrid by QTL mapping in an immortalized $\mathrm{F}_{2}$ population. Theor. Appl. Genet. 120: 333-340.

Trachsel S, Messmer R, Stamp P, Ruta N, et al. (2010). QTLs for early vigor of tropical maize. Mol. Breed. 25: 91-103.

Tuberosa R, Salvi S, Sanguineti MC, Landi P, et al. (2002). Mapping QTLs regulating morpho-physiological traits and yield: case studies, shortcomings and perspectives in drought-stressed maize. Ann. Bot. 89: 941-963.

Voorrips RE (2002). MapChart: software for the graphical presentation of linkage maps and QTLs. J. Hered. 93: 77-78.

Wang S, Basten CJ and Zeng ZB (2010). Windows QTL Cartographer 2.5. Department of Statistics, North Carolina State University, Raleigh. Available at [http://statgen.ncsu.edu/qtlcart/WQTLCart.htm]. Accessed March 10, 2010.

Xiao YN, Li XH, George ML, Li MS, et al. (2005). Quantitative trait locus analysis of drought tolerance and yield in maize in China. Plant Mol. Biol. Rep. 23: 155-165. 